\title{
What evidence exists on the local impacts of energy systems on marine ecosystem services: a systematic map
}

\author{
Eleni Papathanasopoulou*, Ana M. Queirós, Nicola Beaumont, Tara Hooper and Joana Nunes
}

\begin{abstract}
Background: Increasing concentrations of greenhouse gases (GHG) in the atmosphere and its impact on the climate are a pressing concern for governments around the world. Reducing GHG emissions by changing the energy production mix is one option to reach targets being set by international communities. As the implementation of renewable and non-renewable energy infrastructure deployed in marine ecosystems increases, it is not clear how these changes will impact on the marine environment and the ecosystem services it provides. To address this knowledge gap a systematic mapping approach was applied, with three key aims: firstly, to provide an overview of the types of impacts being studied for the offshore components of nuclear, offshore oil \& gas and offshore wind arrays; secondly to demonstrate how these impacts can be translated into ecosystem services; and finally to provide a searchable database of the results.

Methods: Searches for relevant articles were carried out using academic and grey literature databases. A total of 2297 articles were sourced, which were screened using selection criteria that determined which subject populations, exposure types and outcomes were considered relevant. To translate these findings into ecosystem service impacts, the Common International Classification of Ecosystem Services (CICES) and Millennium Ecosystem Assessment frameworks were used to ensure relevance, transparency and replicability.

Results: A total of 50 articles, which equated to 208 studies, spanning four decades since 1970, were selected and coded for the systematic map. Across all energy systems, benthic species were the most studied group. Following this, results then varied by group; marine birds and fish were most prevalent in studies of offshore wind; fish for offshore oil \& gas studies; and pelagic organisms for nuclear. The outcome variables most investigated were changes in population and ecosystem function/process. Of all the ecosystem services associated with the studied impacts, regulating services were investigated most often, due to the large number of studies on benthic organisms. Cultural services, specific to offshore wind, were also prevalent.
\end{abstract}

Discussion: The systematic map provides a searchable database of articles and their relevant studies on the local ecological impacts of marine renewable energies. It has identified a number of potential future areas for primary research; for example, investigating the impacts of decommissioning offshore energy infrastructure on marine habitats and organisms.

Keywords: Biodiversity, Ecosystem functions, Ecological impacts, Marine ecosystem services, Offshore energy systems

\footnotetext{
*Correspondence: elpa@pml.ac.uk

Plymouth Marine Laboratory, Plymouth, UK
} 


\section{Background}

Increasing concentrations of greenhouse gases $\left(\mathrm{GHG}^{1}\right)$ in the atmosphere have been a concern to governments around the world for the last few decades, particularly due to their impacts on global temperatures (the greenhouse effect). Initial efforts to reduce GHG levels led to the UN treaty on climate change [1] followed by the subsequent ratification of the Kyoto Protocol [2] in 1997 by a number of countries and its revision in 2013 [3]. In 2015, the Conference of Parties 21st session (COP21) resulted in a global agreement to keep the average global temperature below $2{ }^{\circ} \mathrm{C}$ above pre-industrial levels. The legally binding COP21 agreement is composed of a number of elements including mitigation activities which proactively reduce GHG emissions to 55 gigatonnes globally by 2030 [4]. Carbon dioxide $\left(\mathrm{CO}_{2}\right)$ released from the burning of fossil fuels for energy is one of the main contributors to the basket of GHG. Replacement of fossil fuels with alternative low-carbon technologies (such as renewables) is one of the strategies to significantly reduce emissions.

Low carbon energy futures require lower carbon energy commodities (such as natural gas and nuclear) and renewable energy commodities (such as wind) to play a more prominent role in a country's energy mix. The UK government is continuing to support action towards this change in fuel mix by setting a target of $15 \%$ for all UK energy consumed by 2020 to be supplied by renewable energy. A significant proportion of this electricity production will come from offshore wind energy (25\% of total projected renewable supply) [5]. Other emerging energy sectors such as wave, tidal and aquatic biomass could also contribute in the future; however, presently these are still fledgling technologies in the testing stage. In the absence of deployments at a commercial scale, there is a lack of evidence on actual environmental impact. Nuclear and natural gas are also seen as major contributors to a lower carbon energy mix. Scenarios in the UK Government's Carbon Plan [6] forecast nuclear energy to contribute between $10-15 \mathrm{GW}$ of power by 2030, reducing the UK's "carbon emissions by between 7 and 14\%" [7]. It is also projected that natural gas will account for at least half of energy used for heating in the UK "well into the 2020s" [6].

Increased use and impact of the marine environment to satisfy these energy supply preferences appears certain, whether it be due to the construction, operation or decommissioning of offshore wind farms, offshore oil \& gas platforms or offshore infrastructure associated with nuclear energy. Research on ecosystems (see for example [8-10]) and human impacts (see for example $[11,12]$ ) has

${ }^{1}$ Greenhouse gases include carbon dioxide, methane and nitrous oxide. shown that there are varied outcomes associated with installing energy provisioning systems into, and next to, the marine environment. However, little or no efforts have been undertaken to compare the potential impacts on the marine environment by different energy systems using a systematic and transparent approach.

Where research has been undertaken into the ecological impacts of energy systems, little has been done to translate these impacts into effects on human wellbeing (but see UK NEA [13]). Ecosystem services (ES) are the benefits people gain from ecosystems, that is the goods and services derived from ecosystems that contribute towards human well-being through, for example, food, equable climate and inspiration. Changes to ecosystems can be translated into their implications for ES, providing another level on which to compare impact on ecosystem services across studies and standardise inputs of increased relevance into policy and management decisions [14]. The translation of ecosystem changes to ecosystem service impacts is facilitated by ES classification systems such as those presented within the UK National Ecosystem Assessment [13], Millennium Ecosystem Assessment [15] and the Common International Classification of Ecosystem Services [16], which define ES according to four main ES groups: provisioning, regulating, supporting and cultural. There is a policy requirement for an evidence base which presents knowledge of the different environmental and social impacts of energy systems within this ES framework. To meet this need, this study aimed to produce a systematic map to catalogue of the type of ecosystem changes being studied across energy systems, and to translate these changes into ecosystem service impacts.

\section{Objectives of the systematic map}

The primary objective of this map is to provide a standardised overview of evidence of the changes in the marine environment resulting from the energy technologies: offshore wind, offshore components of nuclear, offshore gas and offshore oil (with results for oil \& gas combined due to the similarity in infrastructure). These energy systems were chosen as they are currently established in the UK to varying degrees and are expected to continue to contribute significantly to the UK's low carbon energy future. Additionally, the impacts are assigned to different lifecycles stages of the energy systems: construction, operation and decommissioning.

The second objective was to translate identified ecosystem impacts into explicit ecosystem service impacts by using the Common International Classification of Ecosystem Services (CICES) [16] and the Millennium Ecosystem Assessment (MEA) [15] framework. For example, 
the results of ecosystem level impacts were used to derive impacts on the ecosystem services: supporting, provisioning and regulating; while the direct human impacts can be used to derive effects on cultural services [14-16]. The proposed mapping methodology therefore differs from the more frequently observed ES literature which often relies solely on expert judgement for the translation of ecosystem impacts into ES, without the use of standardised protocols, making the process opaque and the ability to compare the findings of different studies challenging.

Finally, this map is intended to improve the understanding of how the provision of ecosystem services will change under different energy provisioning scenarios, and to serve to highlight current knowledge gaps that may help to guide future research.

The primary question used to source the articles was formulated as: "What impacts do the construction, operation and decommissioning of offshore oil \& gas, offshore wind and offshore structures of nuclear installations have on biodiversity, habitat, structure, and function of marine ecosystems, and their relation to human well-being?". The systematic mapping process was then used to code and categorise the articles and their studies according to an array of characteristics described below.

\section{Methods}

This study provides a searchable database of articles catalogued across a variety of variables such as exposure, subject, comparators, outcomes, geographic location and linked to ecosystem services. Coding of the articles in the systematic map enables an analysis of the articles to be undertaken, facilitates their further use by practitioners, identifies knowledge gaps and enables future primary research to be identified.

\section{Search strategy}

The two databases used to source published peerreviewed research were Sciencedirect and Web of Science, and for grey literature was the Aquatic Sciences and Fisheries Abstracts (ASFA) database. The initial list of search terms for the outcome variable built on those from Rilov et al. [17] and Lyons et al. [18] given that the focus of these systematic reviews were to investigate impacts on the wide array of marine organisms and ecosystem processes that concerned the current study. These search term strings were then further developed by combining them with lists of the specific intervention (i.e. energy systems) and subject areas of interest to this study. The final combination of search terms for each of the energy systems that provided the most relevant articles in test searches are shown in Additional file 1.

\section{Study inclusion criteria}

A list of inclusion criteria were agreed by the research team prior to the review to ensure articles selected would address the research objective. The criteria included a range of variables including: subject, exposure, comparators, outcomes and study design [see protocol for details, 19]. As each reviewer was assigned a specific technology it was necessary to ensure that there was a level of agreement between reviewers and consistency in the selection of relevant articles. A Fleiss' kappa test [20] was conducted to measure the degree of agreement between reviewers based on a fixed sub-set of references. Each reviewer was given the same list of randomly selected abstracts from the complete research database and asked to state whether they would include or exclude the article based on the inclusion criteria. The ranges used to identify the degree of interrater reliability were: $0.01-0.20$ represented none to slight agreement, $0.21-0.40$ fair agreement, $0.41-0.60$ moderate agreement, $0.61-0.80$ substantial agreement, and 0.81-1.00 almost perfect agreement [21, 22].

\section{Coding for the systematic map}

Each article retained for the systematic map was read in full and each study contained within the articles were coded using the structure of the primary question, which included exposure, subject and outcome (Additional file 2). For each of these components, a broad grouping structure was agreed to allow a set list of options to be available to the reviewers ensuring comparability across the energy systems. The subject groups considered were: benthic, demersal, pelagic, marine mammals, seabirds and general public. Outcome variables were also grouped according to: species distributions, biodiversity, species richness, community structure, abundance, non-indigenous species, ecosystem function, ecosystem process, recreational use, inspiration, spiritual influence, and human health. Further information providing context to the studies included: geographic location; start and end dates of study; and spatial and temporal scale. Attributes describing the quality of the studies were also included.

The findings of the review were also converted and coded into ecosystem services using the process described in the associated protocol (see reference [19]). The ecosystem service groups used for coding included: provisioning, regulating, supporting and cultural. The stage of the energy system at which the impact on ecosystems and their services were studied were also coded. The variables used for identifying energy system's lifestages are common to lifecycle analyses and include: construction, operation and decommissioning (see Additional file 2). 


\section{Quality assessment of reviewed studies}

Quality criteria were used in this review to score the validity of studies across a range of attributes (see reference [19]). The attribute categories contributed equal importance to the quality assessment and so their scoring totals were evenly weighted. Categorical and additive scoring types were used, and the total data quality score for each study could range from 0 to 100. Details of each of these criteria were extracted from studies read at full text and summed to give a final quality score. This study quality scoring framework is used across all studies reviewed.

\section{Systematic map database}

The findings of the systematic map for offshore wind, offshore oil \& gas and offshore components of nuclear are summarised in a searchable database provided as part of the systematic map protocol. The collation of the results in one table allows an overview of the results to be gained and the translation into ecosystem services to be recorded. It also allows the database to be queried to gain insight into the characteristics of the evidence available to inform the primary question. The results are compared between energy systems and discussed to highlight the types of impacts identified, current gaps in knowledge, and potential implications of future energy mixes.

\section{Results}

\section{Articles sourced and retained for the systematic map}

The search for relevant articles using the selected search strings and search engines returned a total of 2297 articles. The full set of 2297 articles was composed of 691 articles for nuclear (689 peer reviewed articles, 2 grey literature reports), 939 for oil \& gas (913 peer reviewed articles, 29 grey literature reports) and 667 for offshore wind farms (667 peer reviewed articles, 0 grey literature reports) (Fig. 1).

Article selection then proceeded based on the inclusion criteria. A total of 1960 were discarded either because they were duplicates or on review of their titles were judged to be spurious. The total available articles considered in the second filter (abstract review) were 337 (nuclear 85, oil \& gas 200, offshore wind 52). The Fleiss kappa test was carried before proceeding to filter 2 at this stage and returned a result of 0.53 . This result falls within the range of moderate agreement between reviewers. The team discussed the non-agreement to clarify any remaining inconsistencies in understanding and clarification of criteria being used in the selection process. The team then continued with the selection of articles using filter 2 which resulted in a further 199 articles being discarded across all the energy systems. These were split as follows:
40 articles (38 peer reviewed, 2 grey literature), excluded for nuclear; 132 (104 peer reviewed, 28 grey literature), articles for oil \& gas and 27 (27 peer reviewed) articles for offshore wind.

The remaining 138 articles were then read in full resulting in the further exclusion of 88 articles. These articles were excluded for reasons ranging from "no quantifiable data" to "not accessible" and are listed in Additional file 3 by energy type.

Fifty articles were included in the final systematic map composed of 11 articles for nuclear, 22 for oil \& gas and 17 for offshore wind. All articles identified for full article review were available and accessible from on-line journals or hard copies from library archives. The full list of articles included in the systematic map is listed in Additional file 4.

Many of the articles had more than one study as presented in Fig. 1 and seen in the systematic map database (Additional file 5). The total number of studies from which data was extracted totalled 208 studies. These were split: 22 for nuclear, 145 for oil \& gas, and 41 for offshore wind.

The final set of articles used in the systematic review spanned the period 1977-2013 (Fig. 2; column B in Additional file 5). The nuclear and oil \& gas articles included studies from the 1970s and 1990s respectively which is unsurprising giving the presence of nuclear energy from the 1950s and oil \& gas from the late 1800s. Articles focusing on offshore wind energy were more recent, as the first offshore wind farm was only constructed in the 1990s. It is evident from the consistent and increasing presence of these publications over time that investigating the environmental impacts of energy systems is of continuing importance.

\section{Location of articles}

The geographical locations of the studies in the articles are clustered around the main production spots of the three energy systems around the world and associated large marine ecosystems (Fig. 3; column I of Additional file 5). Oil \& gas studies covered the greatest geographical spread ranging from the north Beaufort Sea down to the South Brazil shelf and across to the Laptev Sea, reflecting the proliferation of this fuel around the world. Studies focusing on nuclear energy were concentrated around a latitudinal zone spanning $\mathrm{N} 22^{\circ}$ to $\mathrm{N} 53^{\circ}$, stretching from the NE US Continental Shelf to the Celtic-Biscay Shelf and onto the South and East China Sea. Offshore wind studies were clustered in and closely linked to waters of the Irish Sea, North Sea and Baltic Sea, reflecting Europe's lead in the construction and operation of offshore wind farms. 
Total number of articles identified $\mathbf{n}=\mathbf{2 , 2 9 7}$ (split by energy type)

\begin{tabular}{|c|c|c|}
\hline Nuclear $n=691$ & Oil and gas $n=939$ & Offshore wind $n=667$ \\
\hline $\begin{array}{l}1^{\text {st }} \text { Filter: } 88 \% \\
\text { (606 articles) } \\
\text { excluded as } \\
\text { duplicates and } \\
\text { from title review }\end{array}$ & $\begin{array}{l}1^{\text {st }} \text { Filter: } 78 \% \\
\text { ( } 739 \text { articles) } \\
\text { excluded as } \\
\text { duplicates and } \\
\text { from title review }\end{array}$ & $\begin{array}{l}1^{\text {st }} \text { Filter: } 92 \% \\
\text { ( } 615 \text { articles) } \\
\text { excluded as } \\
\text { duplicates and } \\
\text { from title review }\end{array}$ \\
\hline
\end{tabular}

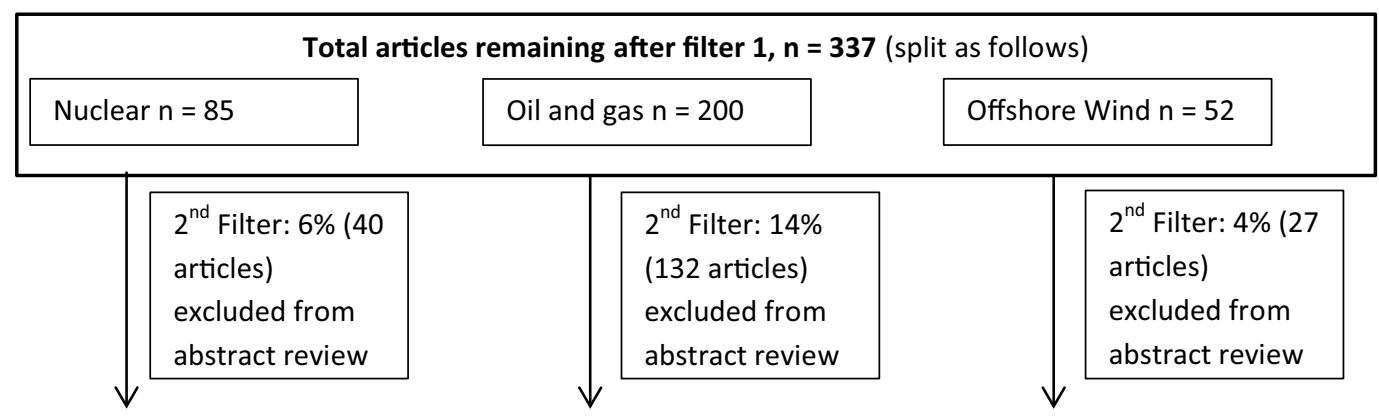

\begin{tabular}{|c|c|c|}
\hline \multicolumn{3}{|c|}{ Total articles remaining $n=138$ (split as follows) } \\
\hline Nuclear $n=45$ & Oil and gas $n=68$ & Offshore Wind $n=25$ \\
\hline $3^{\text {rd }}$ Filter: $5 \%$ (34 articles) & $3^{\text {rd }}$ Filter: $5 \%$ (46 articles) & $3^{\text {rd }}$ Filter: $2 \%$ (8 articles) \\
\hline excluded following full & excluded following full & excluded following full \\
\hline review & review & review \\
\hline No quantifiable data $=4$ & No quantifiable data $=8$ & No quantifiable data $=1$ \\
\hline No relevant & No relevant & No relevant \\
\hline intervention/exposure $=2$ & intervention/exposure $=6$ & intervention/exposure $=3$ \\
\hline No relevant population $=2$ & No relevant population $=11$ & No relevant population $=0$ \\
\hline No relevant comparator $=2$ & No relevant comparator $=9$ & No relevant comparator $=1$ \\
\hline No relevant outcome $=1$ & No relevant outcome $=3$ & No relevant outcome $=3$ \\
\hline Unsuitable study design $=7$ & Unsuitable study design = 9 & Unsuitable study design $=0$ \\
\hline Relevant review $=4$ & Relevant review $=0$ & Relevant review $=0$ \\
\hline Not accessible $=12$ & Not accessible $=0$ & Not accessible $=0$ \\
\hline
\end{tabular}

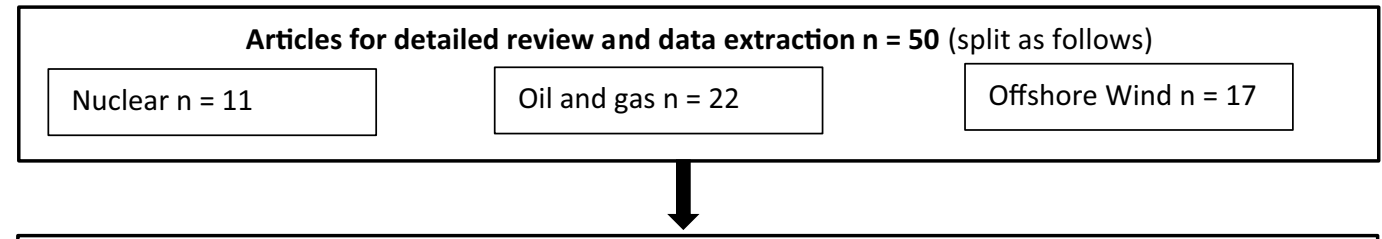

Studies extracted from articles $n=208$ (split as follows)

\begin{tabular}{|l|l|l|}
\hline Nuclear $n=22$ & Oil and gas $n=145$ & Offshore Wind $n=41$ \\
\hline
\end{tabular}

Fig. 1 Detailed schematic of the number of articles included/excluded in the systematic map and their associated studies 


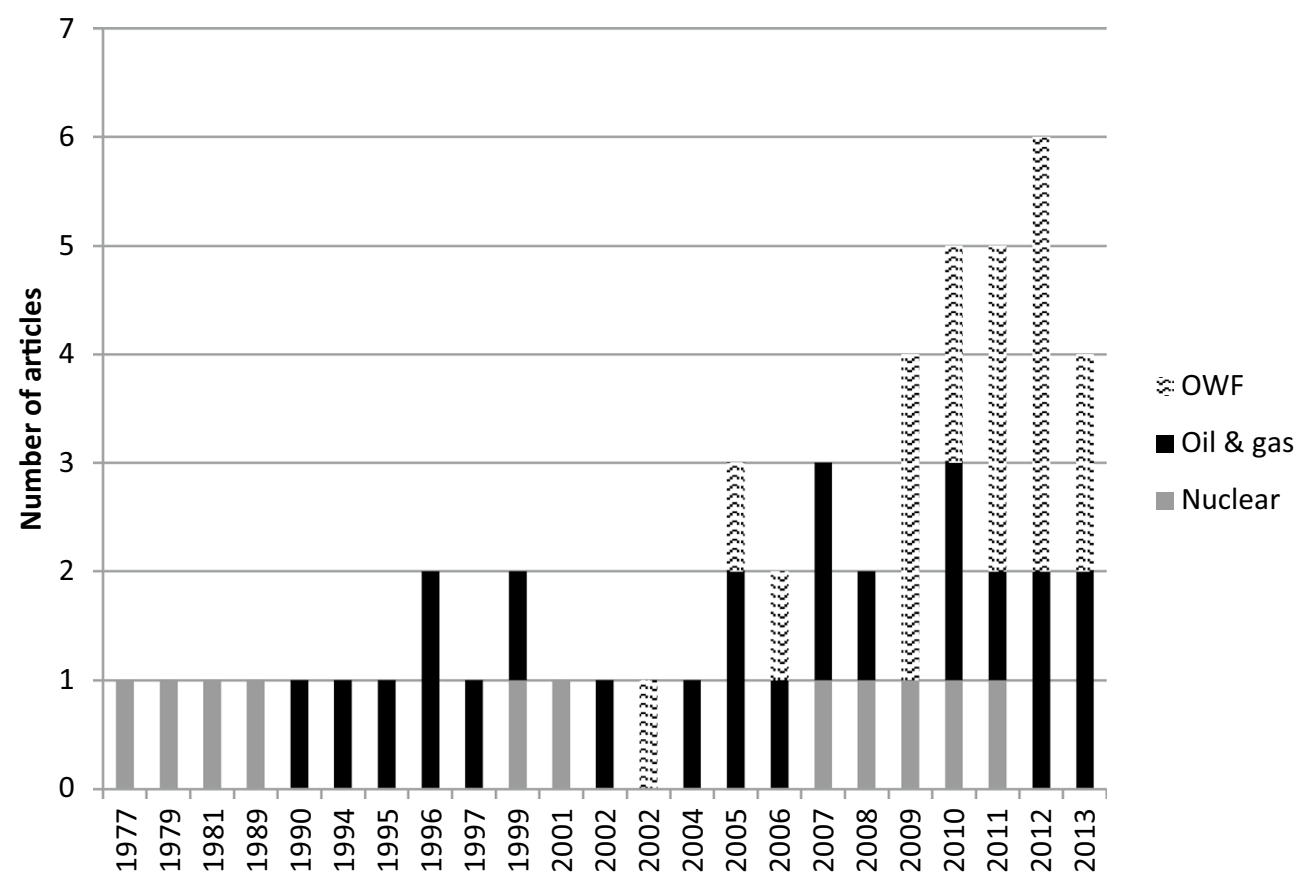

Fig. 2 Year of publication of articles

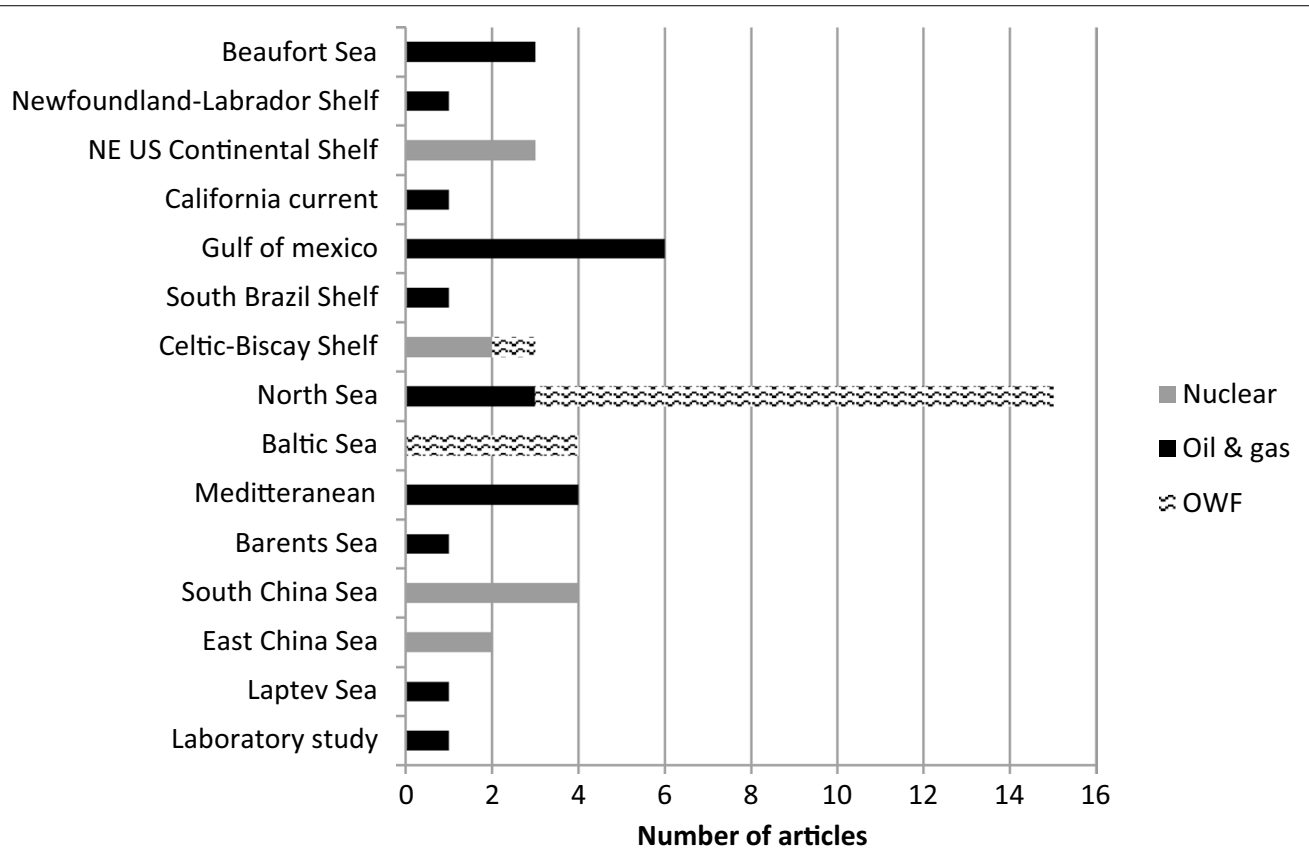

Fig. 3 Number of articles by large marine ecosystem and energy type

\section{Subject details}

The subjects of the reviewed studies for nuclear and oil \& gas appeared to reflect a tradition of benthic impacts, with fewer studies considering the pelagic system (Fig. 4; column E of Additional file 5), possibly because benthic systems may be expected to endure longer lasting effects compared to the more dynamic pelagic communities. Conversely, studies exploring the impacts of offshore 


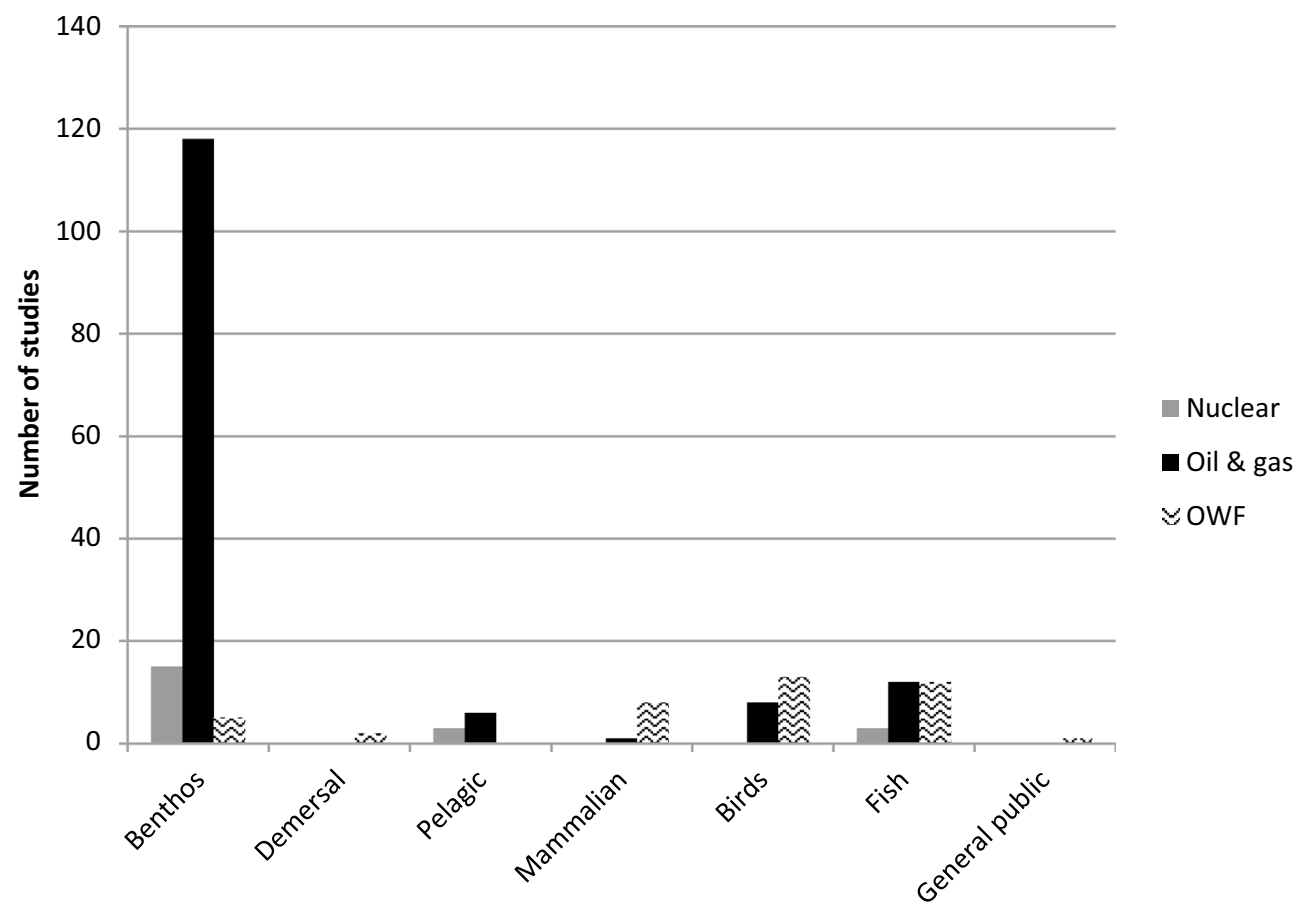

Fig. 4 Studies by main subject type

wind farms had a more specific focus on key and highly mobile species, including marine mammals, birds and fish possibly because wind arrays have a tradition of being considered to cause potential harm to migration routes [23]. Reviewed studies thus seemed to suggest that emphasis is typically placed on evaluating impacts on specific ecosystem components in each case, and that gaps may remain regarding reviews of potential impacts on different ecosystem components for each energy type.

Additionally, by comparing subject type with the lifecycle stage of each energy system (i.e. construction, operation or decommissioning), it was evident that most studies have been focused on the operation phase (Fig. 5; column AC of Additional file 5). Within these studies on operation, $63 \%$ of nuclear, $52 \%$ of oil \& gas and $12 \%$ of offshore wind studies focused on benthic impacts.

\section{Outcome variable}

The outcome variables used in the search terms included thirty different possibilities, but only ten of these were identified in the studies reviewed. The most studied outcome across all energy systems in absolute terms was population change and ecosystem function/process, followed by abundance, biodiversity and community richness (Fig. 6; column $\mathrm{Z}$ of Additional file 5).

However, if these studies are analysed by energy type and subject, then in relative terms ecosystem function/ process was the most common variable investigated particularly in the benthic community, while abundance and population were frequently studied across the majority of subjects (Fig. 7).

\section{Quality of study and design}

Ninety-nine percent of all studies across all energy systems reviewed were designed to collect primary data, and so received the highest score for this attribute. Secondary data made up the remaining 1\% (Fig. 8a; column Q of Additional file 5). The use of fully structured comparator design (i.e. BACI design), which are deemed to provide a more robust basis for impact studies, were restricted to 28 of the studies reviewed (15\% of the total) specifically in oil \& gas and offshore wind farm studies (Fig. 8b; column R of Additional file 5). Forty percent of offshore wind studies were found to follow a BACI design. The next most popular comparator type in absolute terms was the spatial comparator (control-impact, CI), making up $48 \%$ of all studies reviewed for oil \& gas. Nuclear studies were found to have the highest proportion of studies $(45 \%)$ where no spatial or temporal comparator was employed to measure impacts, as studies often reported on data collected over a particular time period during exposure (e.g. operation). This could suggest that impacts are measured through potential changes to the ecosystem during operation, but no real controls are employed. 


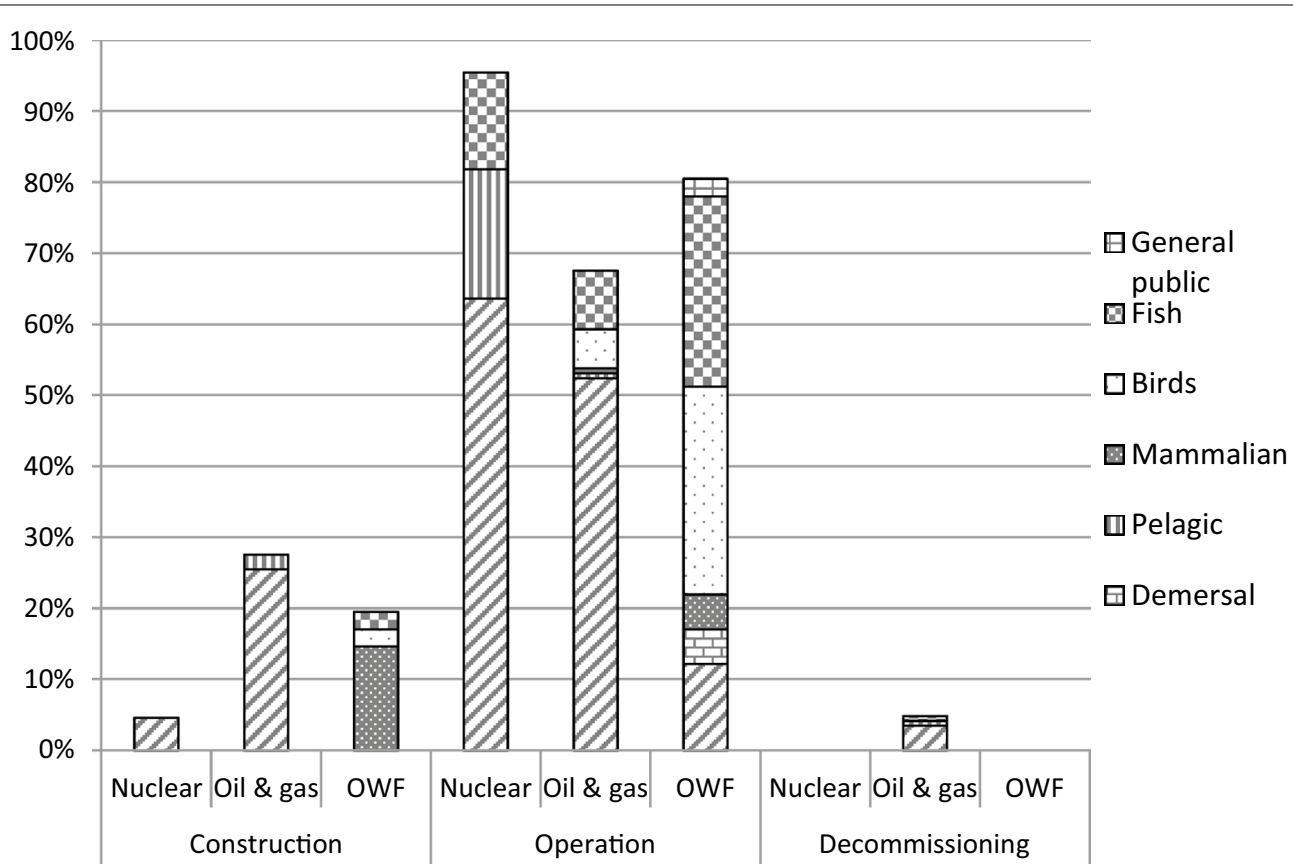

Fig. 5 Subject by energy lifecycle stage

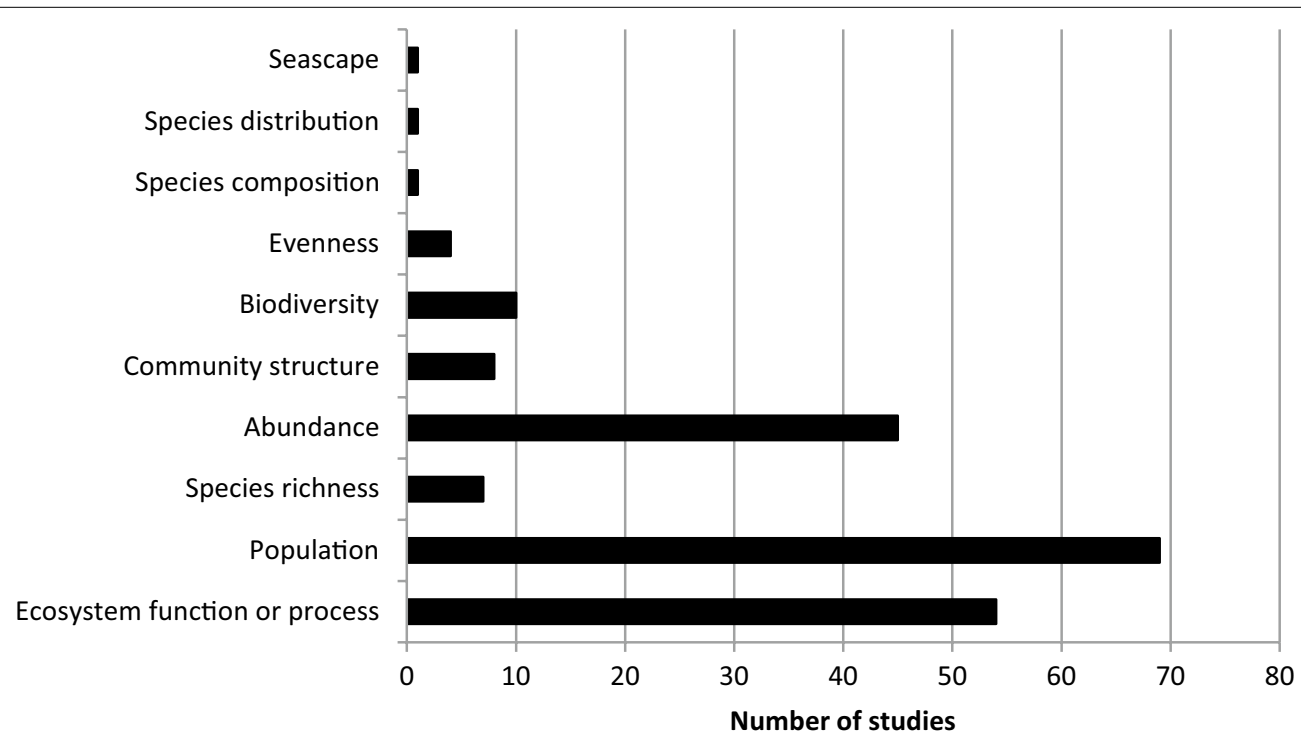

Fig. 6 Outcome variable across all energy types

Replication was the third category considered important in ranking the strength of individual studies (Fig. 8c; column W of Additional file 5). Fifty percent of the studies retained for the systematic map included either temporal or spatial replication, which scored the highest in this category. Over half of all offshore oil \& gas and offshore wind focused studies matched this attributes.

\section{Impact on ecosystem services}

Ecosystem service impacts were not explicitly mentioned or considered in any of the studies reviewed. It was the objective of this systematic map to introduce this extra dimension to allow the studies to be compared in terms of ecosystem services across energy systems. 


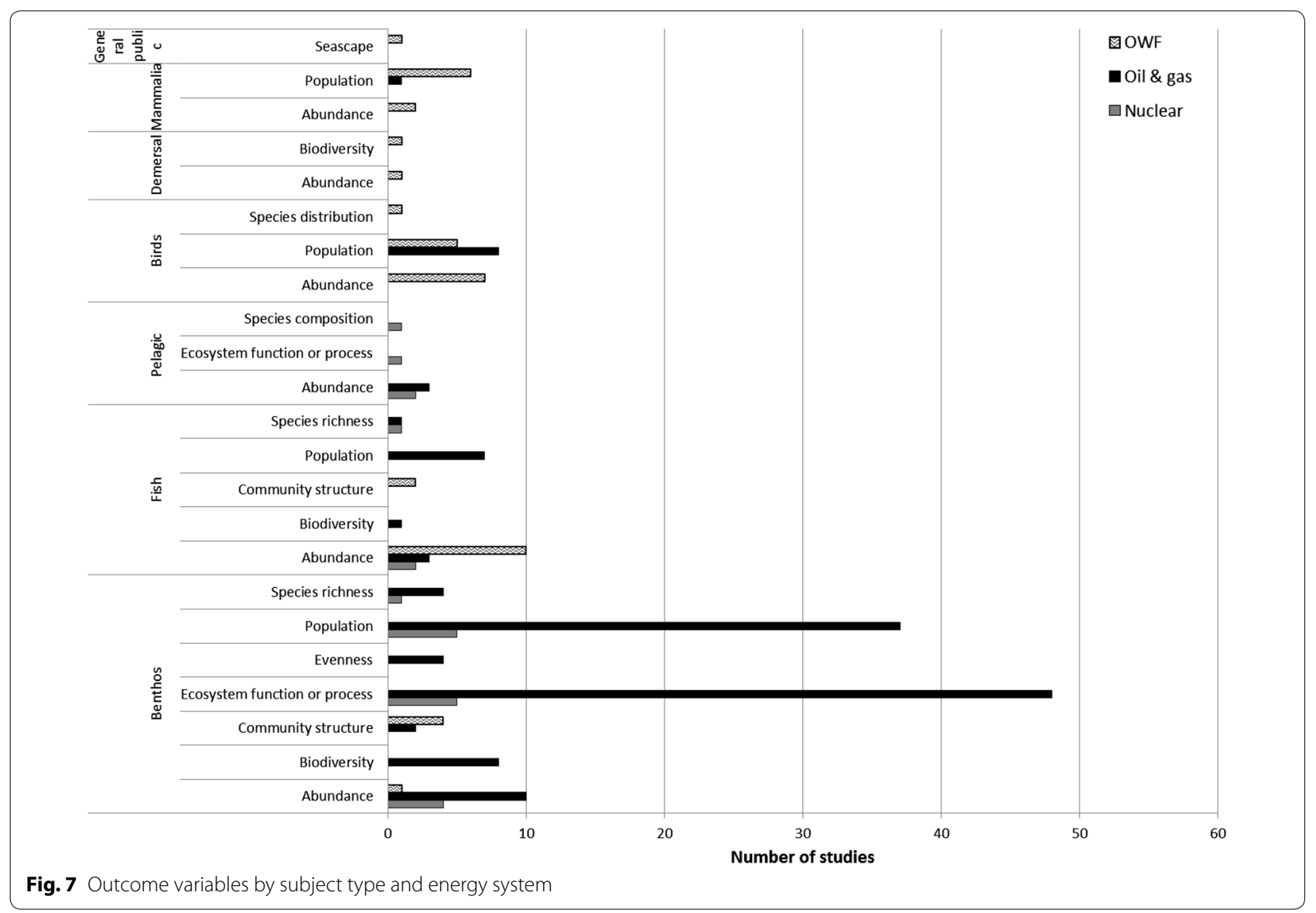

The results show that the species and habitats evaluated in the studies and impacted by offshore energy systems, provide a range of ecosystem services across the provisioning, regulating, supporting and cultural categories. Regulating services were the most commonly associated with the benthic species studied followed by supporting, provisioning and cultural. Across the other species types, cultural services were particularly dominant in the studies of impacts of marine mammals and birds from offshore wind farms. Supporting and provisioning services were also associated with the demersal and pelagic species investigated (Fig. 9; column $\mathrm{AB}$ of Additional file 5).

Translating changes into ecosystem services highlights the opportunity for species other than those traditionally studied to be researched based on the ecosystem services they provide. With the marine environment becoming ever more intensively used, and demands for ecosystem based management, a holistic investigation of a range of species that provide a suite of ecosystem services is required to inform planning and operational decisions.

\section{Discussion}

\section{Key findings}

The results of the systematic map provide an overview of available research investigating the of impacts energy systems on the marine environment.

- A total of 50 articles, which equated to 208 studies, matched the inclusion criteria employed. The articles spanned over almost four decades, beginning in the 1970s and increasing in number as new technologies, such as offshore wind, began to contribute to the energy mix.

- The spatial coverage of studies varied by energy type, reflecting the specific concentration of these technologies in specific large marine ecosystems and geographical areas.

- Benthic species accounted for almost $66 \%$ of subjects in the reviewed studies, followed by fish and birds. This focus seemed to reflect the key ecosystem components expected to be impacted by each energy technology, leaving knowledge gaps pertaining to potential impacts in other ecosystem components. 

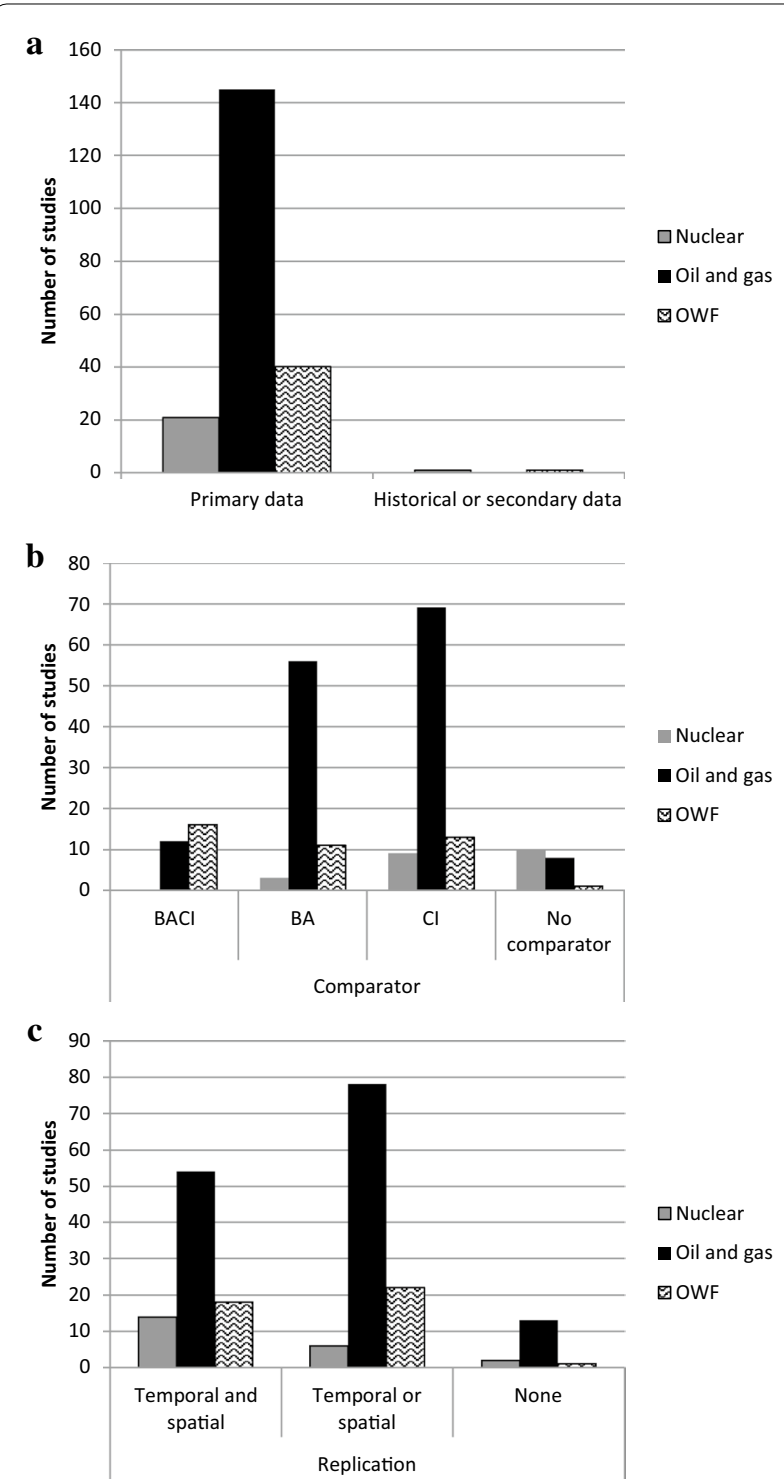

Fig. 8 Quality of studies by a design, b comparator, c replication

- The operational phase was the most commonly studied life-stage of energy systems, followed by construction. Only seven studies considered decommissioning and all were focused on offshore oil \& gas rigs.

- Change in population and ecosystem function/process were the most investigated outcomes over all species groups and technology. However, oil \& gas studies frequently focused also on abundance of benthic species.

- Translation of the impacts to ecosystem services identified that regulating services provided by benthic communities could be expected to be the most frequently impacted, particularly for offshore oil \& gas industry studies. Cultural services dominated offshore wind studies due to the species considered. These outcomes are closely linked to the ecological impact considered in each of the studies, which has been shown to be traditionally focussed on particular species. The results, therefore, cannot be considered to be a representative summary of impacts across ecosystem services for each of the energy systems.

- All the literature retained for the systematic map was sourced from peer reviewed journals and grey literature.

- There were large variations in the quality of the studies ranging from a minimum quality score of 20 (an oil \& gas study) to a maximum of 99 (oil \& gas and offshore wind).

\section{Limitations during searching}

Decisions taken at different stages of the systematic map process will place limitations on the outcome of this study, including choices related to search terms and its development, and the inclusion of all relevant articles in the database, irrespective of the quality score assigned. The objective of this systematic map was to provide an overview of the studies assessing the impact of energy systems on marine ecosystems and ecosystem services. Generic terms were used in the search terms list possibly contributing to the omission of articles with more specific keywords. Extended lists for each of the population search terms might identify additional relevant articles.

Only two peer-reviewed search engines and one grey literature search engine were used due to constraints on resources to conduct the review using a systematic approach. Using alternative search engines, such as the Directory of Open Access Journals, Index to online Thesis, CAB abstracts, Greenfile, NERC Open Research Archive, Scholar Google (for more general web searches), Scirus Dogpile, could capitalise on their search algorithms returning other relevant articles. The articles reviewed were restricted to those returned by the search engines with no additional leads being followed from the articles' bibliographies. It is not possible, therefore, to state the comprehensiveness of the database search and the results should be viewed with this limitation in mind. A future iteration of this review would improve on this by using the bibliographies of the returned articles to identify additional relevant primary articles and involve stakeholders and experts to identify additional potential sources of data.

\section{Limitations to the evidence base}

The quality of the articles reviewed varied considerably, but all, even low-scored articles, were included in the 


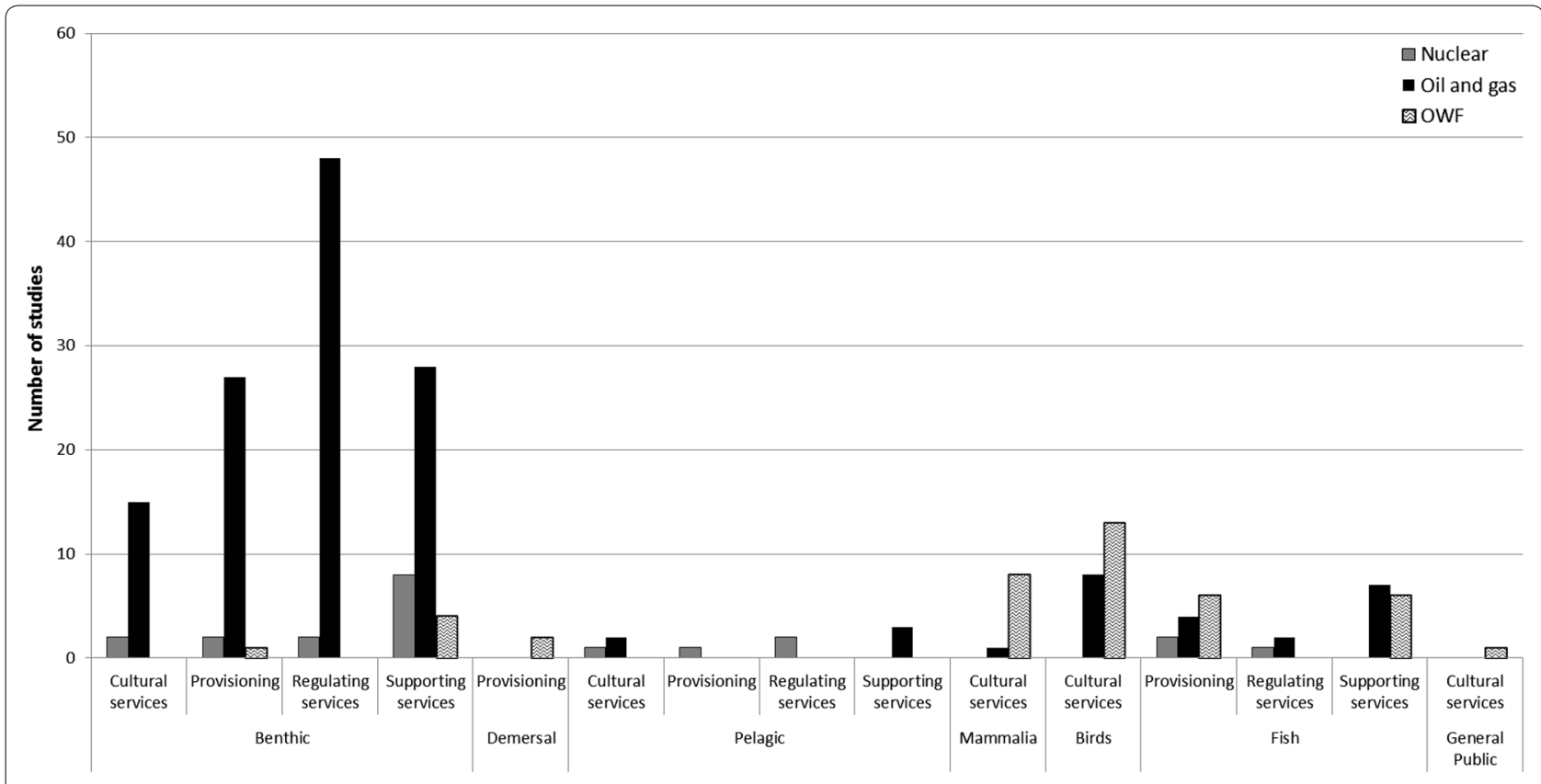

Fig. 9 Ecosystem services mapping

systematic map as all, except one, were peer-reviewed publications. This choice could have influenced the outcomes and recommendations for future research. Analyses of the database by excluding studies, or opting for alternative quality scoring criteria, could provide more robust gap analyses in future studies of this kind, and also highlight different areas of knowledge gaps.

\section{Conclusion}

This systematic map identified and categorised studies contributing to the evidence base to address the primary question: "What are the local impacts of energy systems on marine ecosystem services?". It highlighted that benthic species, population and ecosystem function/process impacts are the most widely studied in offshore oil \& gas, wind and related nuclear research.

\section{Implications for policy and management}

An initial map of the evidence accrued to studies investigating the impact on the marine environment of energy systems has been carried out. It signals to policy and management the types of evidence based research on impacts of species studied for specific low-carbon energies available and lends itself to some preliminary identification of gaps. Given this insight, comprehensive systematic reviews can be undertaken to inform the magnitude and range of impacts and assist in identifying the appropriate mitigation and adaptation actions.

\section{Implications for systematic reviews and primary research} A number of future research areas can be identified and informed from this map. These range from investigation of specific species, range of impacts and spread of geographic locations. Primary research could be carried out to reduce the gap in knowledge on a number of fronts identified by the systematic map. These include (1) understanding the impacts of decommissioning energy infrastructure in the marine environment across species and ecosystem components; (2) increasing the number of studies on the impacts of offshore energy systems on mammals, fish, birds, demersal and pelagic species; and (3) a review of energy system impacts on ecosystem services and their comparison between energy systems.

\section{Additional files}

Additional file 1. Search term development

Additional file 2. Coding of categories used in systematic map database. Additional file 3. Articles excluded at full text.

Additional file 4. Reference included in systematic map database.

Additional file 5. Systematic map database of impact of energy systems on marine ecosystems.

Authors' contributions

$E P, N B, T H, J N, A Q$ contributed to the design of the systematic map, discussion of its components. EP, TH, JN, AQ conducted the mapping activities. EP drafted the manuscript, $T H, J N, A Q, N B$ assisted in the manuscript's refinement. All authors read and approved the final manuscript. 


\section{Acknowledgements}

This research formed part of the programme of the UK Energy Research Centre and was supported by the UK Research Councils under Natural Environment Research Council award NE/G007748/1. AMQ acknowledges support from the Marine Ecosystems Research Programme, which was funded by the Natural Environment Research Council and the Department for the Environment, Food and Rural Affairs of the UK, under award NE/L003279/1.

\section{Competing interests}

The authors declare that they have no competing interests.

Received: 29 March 2016 Accepted: 3 November 2016

Published online: 24 November 2016

\section{References}

1. UNFCCC. United Nations Framework Convention on Climate Change background information. 1992. http://unfccc.int/essential_background/ items/6031.php.

2. UNFCC. Kyoto Protocol. 1997. http://unfccc.int/essential_background/ items/6031.php.

3. UNFCC. Kyoto Protocol. 2014. http://unfccc.int/kyoto_protocol/ items/2830.php. Accessed 07 Mar 16.

4. UNFCC. COP21: Adoption of the Paris Agreement. 2015. https://unfccc. int/resource/docs/2015/cop21/eng/l09r01.pdf. Accessed 07 Mar 16

5. DECC: UK renewable energy road map. London; 2011. https://www. gov.uk/government/uploads/system/uploads/attachment_data/ file/48128/2167-uk-renewable-energy-roadmap.pdf.

6. DECC: The carbon plan—reducing greenhouse gas emissions. London; 2011. https://www.gov.uk/government/publications/ the-carbon-plan-reducing-greenhouse-gas-emissions-2.

7. DECC: Nuclear. London; 2012. http://www.decc.gov.uk/en/content/cms/ meeting_energy/nuclear/nuclear.aspx.

8. Skeate ER, Perrow MR, Gilroy JJ. Likely effects of construction of Scroby Sands offshore wind farm on a mixed population of harbour Phoca vitulina and grey Halichoerus grypus seals. Mar Pollut Bull. 2012;64:872-81.

9. Warwick PE, Cundy AB, Croudace IW, Bains MED, Dale AA. The uptake of iron- 55 by marine sediment, macroalgae, and biota following discharge from a nuclear power station. Environ Sci Technol. 2001;35(11):2171-7.
10. Fabi G, Grati F, Puletti M, Scarcella G. Effects on fish community induced by installation of two gas platforms in the Adriatic Sea. Mar Ecol Prog Ser. 2004;273:187-97.

11. Hooper T, Austen M. Tidal barrages in the UK: ecological and social impacts, potential mitigation, and tools to support barrage planning. Renew Sustain Energy Rev. 2013;23:289-98.

12. Venables D, Pidgeon NF, Parkhill KA, Henwood KL, Simmons P. Living with nuclear power: sense of place, proximity, and risk perceptions in local host communities. J Environ Psychol. 2012;32:371-83.

13. UK National Ecosystem Assessment. The UK National Ecosystem Assessment Technical report. Cambridge: UNEP-WCMC; 2011.

14. DEFRA. Ecosystem Services; 2013. https://www.gov.uk/ ecosystems-services.

15. Millennium Ecosystem Assessment. Ecosystems and human well-being: synthesis. Washington: Island Press; 2005.

16. Haines-Young R, Potschin M. CICES Version 4: response to consultation. London; 2012. http://cices.eu.

17. Rilov G, Mant R, Lyons D, Bulleri F, Benedetti-Cecchi L, Kotta J, Queirós AM, et al. How strong is the effect of invasive ecosystem engineers on the distribution patterns of local species, the local and regional biodiversity and ecosystem functions? Environ Evid. 2012;1(1):1.

18. Lyons DA, Arvanitidis C, Blight AJ, Chatzinikolaou E, Guy-Haim T, Kotta J, Orav-Kotta H, et al. Macroalgal blooms alter community structure and primary productivity in marine ecosystems. Glob Change Biol. 2014;20:2712-24.

19. Papathanasopoulou E, Queirós AM, Beaumont N, Hooper T, Nunes J. What are the local impacts of energy systems on marine ecosystem services: a systematic map protocol. Environ Evid. 2014;3:26.

20. Fleiss JL. Measuring nominal scale agreement among many raters. Psychol Bull. 1971;76(5):378-82.

21. Doerr, E.D., Doerr, V.A.J, Davies, M.J. Does structural connectivity facilitate dispersal of native species in Australia's fragmented terrestrial landscapes? CEE protocol 08-007 (SR 44). Collaboration for Environmental Evidence; 2008 http://www.environmentalevidence.org/SR44.html.

22. McHugh ML. Interrater reliability: the kappa statistic. Biochemia Medica. 2012;22(3):276-82

23. Wang S, Wang S, Smith P. Ecological impacts of wind farms on birds: questions, hypotheses, and research needs. Renew Sustain Energy Rev. 2015;44:599-607.

\section{Submit your next manuscript to BioMed Central and we will help you at every step:}

- We accept pre-submission inquiries

- Our selector tool helps you to find the most relevant journal

- We provide round the clock customer support

- Convenient online submission

- Thorough peer review

- Inclusion in PubMed and all major indexing services

- Maximum visibility for your research

Submit your manuscript at www.biomedcentral.com/submit
Ciomed Central 